\title{
MPMI ACKNOWLEDGMENT OF REVIEWERS
}

The success of Molecular Plant-Microbe Interactions depends on the quality of manuscripts submitted by authors and on the care and competence with which they are reviewed. It is the policy of the Editorial Board to solicit reviews of manuscripts from specialists most qualified to review them. In addition to members of the Editorial Board, the individuals listed below provided constructive critical reviews of one or more manuscripts during the past year. Their names are published here in grateful appreciation for their contributions to the journal.

C. Allen, University of Wisconsin-Madison, U.S.A.

J. Anderson, CSIRO, Wembley, Western Australia, Australia

R. Angelini, University Roma Tre, Rome, Italy

M. Arlat, INRA-CNRS, Castanet Tolosan, France

R. Arredondo-Peter, Universidad Autónoma del Estado de

Morelos, Cuernavaca, México.

B. Bailey, ARS-USDA, Beltsville, MD, U.S.A.

P. Bakker, University of Utrecht, Netherlands

G. Bakkeren, Agriculture \& Agri-Food Canada, BC, Canada

M. Bar-Joseph, Volcani Center, Bet Dagan, Israel

C. Baron, Université de Montréal, QC, Canada

Y. Bashan, University of Arizona, Tucson, AZ, U.S.A.

G. Becard, CNRS Université Paul Sabatier, Castanet-Tolosan, France

A. Becker, University of Freiburg, Germany

A. Bent, University of Wisconsin-Madison, U.S.A.

A. Berry, University of California, Davis, U.S.A.

D. Bertioli, Universidade Católica de Brasília, DF, Brazil

M. K. Bhattacharyya, Iowa State University, IA, U.S.A.

T. Bleve-Zacheo, Istituto per la Protezione delle Piante, CNR, Bari, Italy

A. Block, University of Nebraska, Lincoln, U.S.A.

V. Blok, Scottish Crop Research Institute, Dundee, U.K.

J. Boch, Martin Luther University, Halle, Germany

P. Boevink, Scottish Crop Research Institute, Dundee, U.K.

A. Bogdanove, Iowa State University, Ames, U.S.A.

M. Bolton, USDA-ARS, Fargo, ND, U.S.A.

J.-J. Bono, UMR CNRS UPS 5546, Castanet Tolosan, France

A. Bottin, Université Paul-Sabatier, Castanet-Tolosan, France

K. Bouarab, Sherbrooke University, Sherbrooke, QC, Canada

C. A. Boucher, INRA-CNRS, LIPM, Castanet Tolosan, France

J. E. Bradshaw, Scottish Crop Research Institute, Dundee, U.K.

G. Braus, Georg-August-University Goettingen, Germany

P. Bronstein, USDA-ARS, Ithaca, NY, U.S.A.

B. Broughton, University of Geneva, Switzerland

J. Brown, John Innes Centre, Norwich, U.K.

C. Brown, USDA-ARS, Prosser, Washington, U.S.A.

F. Brunner, ZMBP-Eberhard Karls University, Tuebingen, Germany

D. Buettner, Martin Luther University, Halle, Germany

R. Cameron, McMaster University, Hamilton, Canada

B. Cammue, K. U. Leuven, Heverlee, Belgium

C. Caprari, University of Molise, Pesche, Italy

S. Cartinhour, USDA-ARS, Ithaca, NY, U.S.A.

J. Castillo, Proinpa Foundation, Cochabamba, Bolivia

Y.-K. Chan, Agriculture \& Agri-Food Canada, Ottawa, ON, Canada

J. Chang, Oregon State University, Corvallis, OR, U.S.A.

T. C. Charles, University of Waterloo, Waterloo, ON, Canada

D. Chinchilla, University of Basel, Switzerland

M. Chiurazzi, CNR, Napoli, Italy

S. Chivasa, Durham University, U.K.

A. C. Churchill, Cornell University, Ithaca, NY, U.S.A.
F. Cillo, CNR, Bari, Italy

S. J. Clough, USDA-ARS, Urbana, IL, U.S.A.

G. Coaker, University of California, Davis, U.S.A.

A. Collmer, Cornell University, Ithaca, NY, U.S.A.

P. Constabel, University of Victoria, BC, Canada

B. Cooper, USDA-ARS, Beltsville, MD, U.S.A.

L. Copeland, University of Sydney, NSW, Australia

D. L. Coplin, Ohio State University, Columbus, U.S.A.

S. Coulthurst, University of Dundee, U.K.

W. A. Cowling, The University of Western Australia, Crawley, WA, Australia

K. Craven, Samuel Roberts Noble Foundation, Ardmore, OK, U.S.A.

M. Crespi, CNRS, Gif Sur Yvette, F-91198 France,

J. N. Culver, University of Maryland Biotechnology Institute, College Park, MD, U.S.A.

C. Dardick, Appalachian Fruit Research Station, Kearneysville, WV, U.S.A.

E. L. Davis, North Carolina State University, Raleigh, NC, U.S.A.

M. J. Davis, University of Florida, Homestead, FL, U.S.A.

B. Dawson, University of Florida, Winter Haven, FL, U.S.A.

M. De Bolle, Katholieke Universiteit Leuven, Belgium

D. De Koeyer, Potato Research Centre, Fredericton, New Brunswick, Canada

H. B. Deising, Martin Luther University, Halle, Germany

A. Dellagi, INRA/INA/Université Paris, France

K. J. Denby, University of Warwick, Wellesbourne, U.K.

T. P. Denny, University of Georgia, Athens, GA, U.S.A.

C. Després, Brock University, St. Catharines, ON, Canada

Y. Dessaux, Institut des Sciences du Vegetal, CNRS, Gif-surYvette, France

D. Desveaux, University of Toronto, ON, Canada

M. Dickinson, University of Nottingham, U.K.

R. Dickstein, University of North Texas, Denton, TX, U.S.A.

S. P. Dinesh-Kumar, Yale University, New Haven, CT, U.S.A.

X. S. Ding, Samuel Roberts Noble Foundation, Ardmore, OK, U.S.A.

M. A. Djordjevic, Australian National University, Canberra, ACT, Australia

K. F. Dobinson, Agriculture \& Agri-Food Canada, London, ON, Canada

P. Dodds, CSIRO, Canberra, Australia

N. Donofrio, University of Delaware, Newark, U.S.A.

A. Dorrance, Ohio State University, Wooster, OH, U.S.A.

C. Douglas, University of British Columbia, Vancouver, BC, Canada

R. D'Ovidio, University of Tuscia, Viterbo, Italy

J. Maxwell, Dow, University College, Cork, Ireland

A. Downie, John Innes Centre, Norwich, U.K.

J. Draper, Aberystwyth University, Aberystwyth, U.K.

B. Dumas, CNRS, Université Paul Sabatier, Castanet Tolosan, France 
L. Dunkle, USDA-ARS, West Lafayette, IN, U.S.A.

S. Duplessis, INRA, Champenoux, France

J. Durner, Helmholtz Zentrum München, Germany

D. J. Ebbole, Texas A \& M University, College Station, TX, U.S.A.

L. Eberl, University of Zurich, Switzerland

D. Edwards, University of Queensland, Brisbane, Australia

O. Edwards, CSIRO, Wembley, WA, Australia

R. Eichenlaub, Universität Bielefeld, Germany

A. K.M. Ekramoddoullah, Pacific Forestry Centre, Victoria, BC, Canada

K.-H. Engel, Technische Universität München, Germany

L. Epstein, University of California, Davis, CA, U.S.A.

M. Espinosa-Urgel, CSIC, Granada, Spain

P. van Esse, Wageningen University, Netherlands

T. Eulgem, University of California, Riverside, U.S.A.

S. Eveillard, INRA, Villenave d'Ornon, France

D. Expert, CNRS, Paris, France

M. Fagard, INRA, Paris, France

M. Farman, University of Kentucky, Lexington, U.S.A.

S. K. Farrand, University of Illinois at Urbana-Champaign, U.S.A.

F. Favaron, University of Padova, Legnaro, Italy

B. Favery, UMR INRA, Sophia-Antipolis, France

G. Felix, Botanisches Institut, Basel, Switzerland

G.-A. Fernando, UPM, Madrid, Spain

S. Ferrari, Università di Roma "La Sapienza", Rome, Italy

N. Ferrol, Estacion Experimental Del Zaidon, Granada, Spain

L. Field, Rothamsted Research, Harpenden, U.K.

T. Finan, McMaster University, Hamilton, ON, Canada

R. Flores, CSIC/Universidad Politécnica de Valencia Valencia, Spain

P.R. Fobert, NRC Plant Biotechnology Institute, Saskatoon, Canada

A. Foster, Institute for Biotechnology and Drug Research, Kaiserslautern, Germany

J. Frugoli, Clemson University, Clemson, SC, U.S.A.

W. Fry, Cornell University, Ithaca, NY, U.S.A.

Z. Q. Fu, University of Nebraska-Lincoln, U.S.A.

I. Fudal, INRA, Versailles Cedex, France

C. Fuqua, Indiana University, Bloomington, U.S.A.

E. Galiana, INRA-Université Nice, Antibes Cedex, France

A. Gal-On, The Volcani Center, Bet Dagon, Israel

P. Gamas, INRA/CNRS, LIPM, Castanet Tolosan, France

J. A. García, Centro Nacional de Biotecnología, Madrid, Spain

D. G. Gardiner, CSIRO, St Lucia, Queensland, Australia

W. Gassmann, University of Missouri, Columbia, U.S.A.

E. Gaulin, Université Toulouse, Castanet-Tolosan, France

K. Geider, Research Centre for Agriculture and Forestry,

Dossenheim, Germany

S. B. Gelvin, Purdue University, West Lafayette, IN, U.S.A.

L. Gentzbittel, INP-ENSA Toulouse, Castanet-Tolosan, France

S. A. Ghabrial, University of Kentucky, Lexington, U.S.A.

R. Gilbertson, University of California, Davis, U.S.A.

A. Gioti, University College London, U.K.

J. Glazebrook, University of Minnesota, St. Paul, U.S.A.

S. E. Gold, University of Georgia, Athens, U.S.A.

D. Gonsalves, USDA, Hilo, Hawaii, U.S.A.

S. Goodwin, USDA-ARS, Purdue University, West Lafayette, IN, U.S.A.

P. Goodwin, University of Guelph, ON, Canada

M. Göttfert, Technische Universität Dresden, Germany

A. Goverse, Wageningen University, Netherlands

S. R. Grant, University of North Carolina, Chapel Hill, U.S.A.

M. Grant, University of Exeter, Devon, U.K.

J. Greenberg, University of Chicago, IL, U.S.A.

G. W. Griffith, Aberystwyth University, Wales, U.K.
B. Gronenborn, CNRS, Gif sur Yvette, France

F. Grundler, BOKU University, Vienna, Austria

M. Guilhabert-Goya, Agraquest, Davis, CA, U.S.A.

M. Guo, University of Nebraska, Lincoln, U.S.A.

D. Guttman, University of Toronto, ON, Canada

D. Halterman, University of Wisconsin-Madison, U.S.A.

D. Hannapel, Iowa State University, Ames, U.S.A.

A. Hartmann, GSF-Research Center for Environment and Health, Neuherberg, Germany

P. M. Hasegawa, Purdue University, West Lafayette, IN, U.S.A.

S. Y. He, Michigan State University, East Lansing, U.S.A.

J. Hibberd, University of Cambridge, U.K.

H. Hoch, Cornell University, Geneva, NY, U.S.A.

M. Höfte, Ghent University, Belgium

S. Holaday, Texas Tech University, Lubbock, TX, U.S.A.

M. M. Holsters, Ghent University, Belgium

E. B. Holub, University of Warwick, Wellesbourne, U.K.

P. Hooykaas, Institute of Biology Leiden, Netherlands

B. Horwitz, Technion, Haifa, Israel

B. Howlett, University of Melbourne, Parkville, VIC, Australia

R. Huckelhoven, Technical University of Munich, Freising, Germany

M. Humphry, Max Planck Institute for Plant Breeding, Cologne, Germany

R. Hussey, University of Georgia, Athens, U.S.A.

M. Hyakumachi, Gifu University, Japan

R. Innes, Indiana University, Bloomington, IN, U.S.A.

S. Ito, Yamaguchi University, Japan

Y. Itoh, University of Tottori, Japan

R. Jackson, University of Reading, U.K.

G. Jander, Boyce Thompson Institute, Ithaca, NY, U.S.A.

S. Jansson, Umea Plant Science Centre, Sweden

Y. Jia, USDA ARS, Stuttgart, AR, U.S.A.

H. Jin, University of California, Riverside, U.S.A.

E. Johansen, University of Aarhus, Frederiksberg, Denmark

E. G. Johnson, Cornell University, Ithaca, NY, U.S.A.

Scott Johnson, Scottish Crop Research Institute, Invergowie, Dundee, U.K.

R. Jones, USDA-ARS, Beltsville, MD, U.S.A.

K. M. Jones, Florida State University, Tallahassee, U.S.A.

J. Jones, Scottish Crop Research Institute, Dundee, U.K.

D. Jones, Research School of Biological Sciences, Canberra, ACT, Australia

M. H. A. J., Joosten, Wageningen University, Netherlands

K. Jung, University of California, Davis, U.S.A.

A. Kachroo, University of Kentucky, Lexington, U.S.A.

M. Kahn, Washington State University, Pullman, U.S.A.

I. Kaloshian, University of California, Riverside, U.S.A.

S. Kamoun, John Innes Centre, Norwich, U.K.

S. Kang, Penn State, University Park, PA, U.S.A.

N. Kav, University of Alberta, Edmonton, Canada

Y. Kawazu, National Institute of Vegetable and Tea Science,

Mie, Japan

K. Kazan, CSIRO, Brisbane, Queensland, Australia

D. Keating, Loyola University-Chicago, Maywood, IL, U.S.A.

E. Kellogg, University of Missouri-St Louis, MO, U.S.A.

B. Kemmerling, University of Tübingen, Germany

A. Kiba, Kochi University, Nankoku, Japan

D. Kliebenstein, University of California, Davis, U.S.A.

R. Koebnik, Institut de recherche pour le développement, Montpellier, France

M. V. Kolomiets, Texas A\&M University, College Station, TX, U.S.A.

H. Koltai, Volcani Center, Bet Dagan, Israel

Y. Kubo, Kyoto Prefectural University, Kyoto, Japan

B. N. Kunkel, Washington University, St. Louis, MO, U.S.A. 
H. Küster, Bielefeld University, Germany

C. Lacomme, University of Edinburgh, U.K.

T. Lahaye, Martin-Luther University Halle-Wittenberg,

Germany

L. Lanfranco, Università di Torino, Italy

P. Lariguet, University of Geneva, Switzerland

E. Lauber, INRA-CNRS, LIPM, Castanet-Tolosan, France

C. Lawrence, Virginia Polytechnic Institute and State

University, Blacksburg, VA, U.S.A.

M.-H. Lebrun, CNRS, Lyon, France

K. Lehto, University of Turku, Turku, Finland

P. Lemanceau, INRA/Université de Bourgogne, Dijon, France

D. LeRudulier, UMR CNRS-INRA-Universite de Nice-Sophia Antipolis, France

E. Lewinsohn, Agricultural Research Organization, Ramat Yishay, Israel

M. G. Lewsey, University of Cambridge, U.K.

A. Lichter, Volcani Center, Bet Dagan, Israel

C. J. Lilley, University of Leeds, U.K.

N.-S. Lin, Institute of Plant and Microbial Biology, Academia Sinica, Taipei, Taiwan

G. J. Loake, University of Edinburgh, U.K.

J. Loper, USDA ARS, Corvallis, OR, U.S.A.

J. A. Lucas, Rothamsted Research, Harpenden, U.K.

J. Ludwig-Müller, Technische Universität, Dresden, Germany

E. Lupotto, Istituto Sperimentale per la Cerealicoltura,

Bergamo, Italy

D. Mackey, Ohio State University, Columbus, U.S.A.

R. Mahalingam, Oklahoma State University, Stillwater, U.S.A.

J. W. Mansfield, Imperial College at Wye, Ashford, U.K.

D. Manter, USDA ARS, Fort Collins, CO, U.S.A.

F. M. Martin, INRA, Champenoux, France

G. Martin, Boyce Thompson Institute, Ithaca, NY, U.S.A.

U. Mathesius, Australian National University, Canberra,

Australia

B. Mauch-Mani, University of Neuchatel, Switzerland

A. Maule, John Innes Centre, Norwich, U.K.

W. H. McDonald, Oak Ridge National Laboratory, Oak Ridge, TN, U.S.A.

J. M. McDowell, Virginia Polytechnic Institute and State University, Blacksburg, U.S.A.

H. McIntyre, University of Otago, Dunedin, New Zealand

M. Melotto, University of Texas at Arlington, TX, U.S.A.

T. Mengiste, Purdue University, West Lafayette, IN, U.S.A.

R. W. Michelmore, University of California, Davis, U.S.A.

J. Michiels, Katholieke Universiteit Leuven, Belgium

C. Michielse, University of Amsterdam, Netherlands

S. Miller, Biomerit Research Centre, Cork, Ireland

J. Milner, University of Glasgow, Scotland, U.K.

K. Minamisawa, Tohoku University, Sendai, Miyagi, Japan

M. G., Mitchum, University of Missouri, Columbia, U.S.A.

B. Moerschbacher, Westfälische Wilhelms-Universität Münster Germany

P. Moffett, Boyce Thompson Institute, Ithaca, NY, U.S.A.

A. Molina, Universidad Politecnica Madrid, Spain

P. Morris, Bowling Green State University, OH, U.S.A.

G. Mouille, INRA, Versailles, France

M. B. Mudgett, Stanford University, Stanford, CA, U.S.A.

L. Mur, University of Wales, Aberystwyth, U.K.

A. Murphy, Cambridge University, U.K.

J. Murray, The Samuel Roberts Noble Foundation, Ardmore, OK, U.S.A.

R. Musser, Western Illinois University, Macomb, IL, U.S.A.

K. Mysore, Samuel Roberts Noble Foundation, Ardmore, OK, U.S.A.

W. H. Nasser, INSA, Villeurbanne, France

A. Niebel, INRA-CNRS, LIPM, Castanet-Tolosan, France
R. E. Niks, Wageningen University, Netherlands

G. Noctor, Université Paris XI sud, Orsay, France

L. Noël, INRA/CNRS, LIPM, Castanet-Tolosan, France

T. Nürnberger, University Tübingen, Tübingen, Germany

D. Nuss, University of Maryland, Rockville, MD, U.S.A.

I. Oberplicher, University of Karlsruhe, Germany

Y. Okon, Hebrew University, Rehovot, Israel

T. Ott, University of Munich, Germany

P. F., Palukaitis, Scottish Crop Research Institute, Dundee, U.K.

P. W. Pare, Texas Tech University, Lubbock, TX, U.S.A.

J. E. Parker, Max Planck Institute for Plant Breeding, Cologne, Germany

M. Parniske, Ludwig Maximilian University, Munich, Germany

K. Pawlowski, Stockholm University, Sweden

G. Payne, North Carolina State University, Raleigh, NC, U.S.A.

C. Pieterse, Utrecht University, Netherlands

A. L.T. Powell, University of California, Davis, U.S.A.

J. Prell, John Innes Centre, Norwich, U.K.

B. Pruess, North Dakota State University, Fargo, U.S.A.

A. Pugin, UMR Université de Bourgogne INRA/CNRS, Dijon, France

B. Py, CNRS, Marseille, France

R. Raina, Syracuse University, NY, U.S.A.

C. Rampitsch, Agriculture and Agri-Food Canada, Winnipeg, MB, Canada

P. Ratet, Institut des Sciences du Végétal, Gif sur Yvette, France

J. Rathjen, Sainsbury Laboratory, Norwich, U.K.

M. Ravensdale, Scottish Crop Research Institute, Dundee, U.K.

C. Rey, University of Witwatersrand, Johannesburg, Republic of South Africa

P. Reymond, University of Lausanne, Switzerland

F. Rezzonico, Agroscope ACW Changins-Wädenswil, Switzerland

S. Robatzek, Max-Planck-Institute for Plant Breeding, Cologne, Germany

J. Robb, University of Guelph, ON, Canada

D. Roberts, University of Tennessee, Knoxville, U.S.A.

F. Robertson, University of Cambridge, U.K.

D. Roby, LIPM INRA/CNRS, Castanet-Tolosan, France

M. Rocha-Sosa, UNAM, Cuernavaca, Morelos, Mexico

P. Rodriquez-Palenzuela, ETS de Ingenieros Agrónomos de Madrid, Spain

J. A. Rollins, University of Florida, Gainesville, U.S.A.

P. C. Ronald, University of California, Davis, U.S.A.

M. J. Roossinck, The Samuel Roberts Noble Foundation, Ardmore, OK, U.S.A.

M.-N. Rosso, National Institute for Agricultural Research, Sophia Antipolis, France

H. C. Rowe, University of California, Davis, U.S.A.

C. M. Rush, Texas AgriLife Research, Bushland, TX, U.S.A.

M. Sadowsky, University of Minnesota, St. Paul, U.S.A.

K. Saeki, Nara Women's University, Nara, Japan

B. San Segundo, Consorcio CSIC-IRTA, Barcelona, Spain

F. Sanchez, Instituto de Biotecnología, UNAM, Cuernavaca, Mexico

I. Sanders, University of Lausanne, Switzerland

R. Sandrock, Q Therapeutics, Inc., Salt Lake City, UT, U.S.A.

W. Schäfer, University of Hamburg, Germany

U. Schaffrath, RWTH Aachen University, Germany

P. Schenk, University of Queensland, St Lucia, Australia

N. L. Schlaich, RWTH Aachen University, Aachen, Germany

H. B. Scholthof, Texas A\&M University, College Station, 
U.S.A.

H.-j. Schoonbeek, University of Fribourg, Switzerland

B. Scott, Massey University, Palmerston North, New Zealand

J. S. Scott-Craig, Michigan State University, East Lansing, U.S.A.

G. Sessa, Tel Aviv University, Israel

R. Shepherd, University of California, Berkeley, U.S. A.

V. Shevchik, CNRS/INSA/UCB, Université de Lyon I, France

K. Shirasu, RIKEN Plant Science Center, Yokohama, Japan

P. Simoneau, Université Angers, France

K. B, Singh, CSIRO, Perth, Australia

I. E, Somssich, Max-Planck-Institut für Züchtungsforschung, Cologne, Germany

R. Sonti, Centre for Cellular and Molecular Biology, Hyderabad, India

G. Stacey, University of Missouri, Columbia, U.S.A.

P. Staswick, University of Nebraska, Lincoln, U.S.A.

L. Stein, University of California, Riverside, U.S.A.

I. Stergiopoulos, Wageningen University, Netherlands

J. Stougaard, University of Aarhus, Aarhus, Denmark

D. Strack, Leibniz Institute of Plant Biochemistry, Halle, Germany

K. Subramaniam, Indian Institute of Technology, Kanpur, India.

J. T. Sullivan, University of Otago, Dunedin, New Zealand

G. W. Sundin, Michigan State University, East Lansing, U.S.A.

R. Sunkar, Oklahoma State University, Stillwater, U.S.A.

A. Suzuki, Saga University, Saga, Japan

R. Tajima, Tohoku University, Ohsaki, Miyagi, Japan

J. Takabayashi, Kyoto University, Kawaguchi, Japan.

H. Takahashi, Tohoku University, Sendai, Miyagi, Japan

F. Takken, University of Amsterdam, Netherlands

W. Tameling, Wageningen University, Netherlands

M. Tepfer, ICGEB Biosafety Outstation, Ca' Tron di Roncade, Italy

M. Teplitski, University of Florida, Gainesville, U.S.A.

R. Thilmony, USDA-ARS, Albany, CA, U.S.A.

H. Thordal-Christensen, University of Copenhagen,

Frederiksberg C, Denmark

S. Timmusk, Uppsala BioCenter, Uppsala, Sweden

M. A. Torres, CBGP UPM-INIA, Pozuelo de Alarcon, Madrid, Spain

I. K. Toth, Scottish Crop Research Institute, Dundee, U.K.

M. Tsagris, University of Crete, Heraklion, Greece

S. Tsuge, Kyoto Prefectural University, Japan

P. Tudzynski, Westfälische Wilhelms-Universität Münster, Germany

B. M. Tyler, Virginia Polytechnic Institute and State University, Blacksburg, VA, U.S.A.

T. Tzfira, University of Michigan, Ann Arbor, MI, U.S.A.
R. Ugalde, UNSAM, San Martín, Buenos Aires, Argentina

S. R. Uppalapati, The Samuel Roberts Noble Foundation, Ardmore, OK, U.S.A.

P. E. Urwin, University of Leeds, U.K.

L. Vaillancourt, University of Kentucky, Lexington,, U.S.A.

B. Valent, Kansas State University, Manhattan, U.S.A.

F. Van Gijsegem, INRA, Paris, France

S. van Hijum, University of Groningen, Netherlands

J. van Kan, Wageningen University, Netherlands

D. Van Sanford, University of Kentucky, Lexington, U.S.A.

C. Vance, University of Minnesota, St Paul, MN, U.S.A.

E. Vandelle, Università degli Studi di Verona, Verona, Italy

V. Venturi, ICGEB, Trieste, Italy

M. Viaud, INRA, Versailles, France

R. T. Voegele, University of Konstanz, Germany

A. A. Vojnov, Centro de Ciencia y Tecnología Dr. Cesar Milstein, Buenos Aires, Argentina

J. Vossen, Wageningen University, Netherlands

F. Waller, Justus-Liebig Universität Giessen, Germany

J. Walsh, Warwick University, U.K.

D. Walters, Scottish Agricultural College, Edinburgh, U.K.

T. Wang, John Innes Centre, Norwich, U.K.

D. Wendehenne, University of Burgundy, Dijon, France

F. F. White, Kansas State University, Manhattan, U.S.A.

K. A. White, York University, Toronto, Canada

F. F. White, Kansas State University, Manhattan, U.S.A.

S. A. Whitham, Iowa State University, Ames, U.S.A.

P. Williams, USDA-ARS, Mississippi State University, U.S.A.

R. Wilson, University of Exeter, U.K.

R. P. Wise, Iowa State University, Ames, U.S.A.

F. Wisniewski-Dyé, Université Lyon 1, Villeurbanne, France

T. Wolpert, Oregon State University, Corvallis, U.S.A.

Liming Xiong, Donald Danforth Plant Science Center, St. Louis, MO, U.S.A.

P. Xu, Samuel Roberts Noble Foundation, Ardmore, OK, U.S.A.

J.-R. Xu, Purdue University, West Lafayette, IN, U.S.A.

Y. Yang, Pennsylvania State University, University Park, U.S.A.

B. Yang, Iowa State University, Ames, U.S.A.

H. Yoshioka, Nagoya University, Aichi, Japan

C. Yost, University of Regina, Saskatoon, Canada

J. Zeier, Julius-von-Sachs-Institute for Biological Sciences, Würzburg, Germany

L. Zhang, Institute of Molecular and Cell Biology, Singapore

Y. Zhang, Samuel Roberts Noble Foundation, Ardmore, OK, U.S.A.

L. Zhang, Institute of Molecular and Cell Biology, Singapore

J. Zhou, National Institute of Biological Sciences, Beijing, China

C. Zipfel, Sainsbury Laboratory, Norwich, U.K. 